\title{
TAGUNG
}

\section{Von der Quelle bis zur Mündung: der Donauraum als strategische Region für die Europäische Union}

\author{
Marcel Krönert*
}

Mit der Übernahme der EU-Ratspräsidentschaft durch Ungarn im Januar 2011 wurde die „Strategie der Europäischen Union für den Donauraum“ (Donaustrategie) zu einer der Prioritäten der aktuellen EU-Politik. Die Strategie und der Aktionsplan zur Umsetzung des neuen Politikinstruments werden im Laufe der ungarischen EU-Ratspräsidentschaft in der ersten Jahreshälfte 2011 verabschiedet und betreffen die 14 Staaten im Einzugsgebiet der Donau, von denen acht Mitgliedstaaten der Europäischen Union ${ }^{1}$ und sechs Staaten keine EU-Mitglieder ${ }^{2}$ sind. Damit inkorporiert die Strategie die Idee der Makroregion, welche auch in der EU-Regionalpolitik für den Ostseeraum die Grundlage bildet.

Die Europäische Kommission verfolgt mit der EU-Donaustrategie anspruchsvolle Ziele: Verbesserung der Infrastruktur und Transportwege, Umweltschutz, wirtschaftliche Entwicklung, (Energie-) Sicherheit und Intensivierung von Forschung und Bildung werden angestrebt. Um das Potenzial der EU-Donaustrategie im Speziellen und das des makroregionalen Governance-Ansatzes im Allgemeinen zu erörtern, veranstalteten die Europäische Akademie Berlin, der Arbeitskreis Europäische Integration e.V. und das Europa Zentrum BadenWürttemberg die dreitägige Konferenz. ${ }^{3}$

Im ersten Tagungsbeitrag ging es um die Besonderheit des von der Europäischen Union

\section{Die Donaustrategie der Europäischen Union}

Tagung der Europäischen Akademie Berlin, des Europa Zentrums Baden-Württemberg und des Arbeitskreises Europäische Integration e.V.

Mit Unterstützung der Robert Bosch Stiftung Berlin, 17.-19. Januar 2011

\section{Begrüßung}

Prof. Dr. Eckart D. STRATENSCHULTE, Leiter, Europäische Akademie Berlin

Florian H. SETZEN, M.A./M.E.S., Direktor, Europa Zentrum Baden-Württemberg, Stuttgart

Was ist eine „Strategie“ in Abgrenzung zu einer ,Politik“" - und ist das wichtig?

Prof. Dr. Dr. h.c. Werner WEIDENFELD, Direktor, Centrum für angewandte Politikforschung; Ludwig-Maximilians-Universität München

Dreimal ,nein“ und dennoch erfolgreich? Erste Ergebnisse der Ostseestrategie

Dr. Carsten SCHYMIK, Stiftung Wissenschaft und Politik, Berlin

Wie man Politik und Geschichte, Natur und Kultur erfahren kann: Der Iron-Curtain-Trail entlang der Donau

Michael CRAMER, MdEP, Mitglied des Ausschusses für Verkehr und Fremdenverkehr des Europäischen Parlaments

* Marcel Krönert, Diplomand im Fach Politikwissenschaft an der Freien Universität Berlin.

1 Bulgarien, Deutschland, Österreich, Rumänien, die Slowakische Republik, Slowenien, die Tschechische Republik und Ungarn.

2 Bosnien und Herzegowina, Kroatien, die Republik Moldau, Montenegro, Serbien und die Ukraine.

3 Als Ergebnis der Tagung ist im Berliner Wissenschaftsverlag eine Buchpublikation erschienen: Eckart D. Stratenschulte/Florian Setzen: Der europäische Fluss: Die Donau und ihre Regionen als Strategieraum, Berlin 2011 . 
verwendeten Governance-Instruments. Ausgehend von den in der Politikwissenschaft genutzten Definitionen von Politik beschrieb Werner Weidenfeld zunächst die Charakteristika, die das gegenwärtige Erscheinungsbild von Politik prägten. $\mathrm{Zu}$ diesen gehörten die Medialisierung und die Globalisierung, der technologische Wandel sowie die mit Letzterem verbundene Beschleunigung von Informationsflüssen. In der Grundstruktur sei Politik damit heute mehr denn je eine Sphäre, in der es um die Bewältigung des Augenblicks und die Machttechnik des Moments gehe. Dieser Zustand der situativen Hektik führe in Verbindung mit den neuen Risikokalkülen, die sich unter anderem durch den geopolitischen Paradigmenwechsel von 1990 ergeben hätten, zu einer Orientierungs- und Vertrauenskrise. Eine Aufgabe von Politik sei es, Vertrauen zu schaffen und den vorhandenen Vertrauensvorschuss zu sichern, in dem auch nicht-situative Probleme gelöst werden. Dazu eigne sich die langfristig angelegte, kalkulierende Rationalität einer Strategie. Das Wirkungsprinzip einer Strategie bestehe darin, zwischen den beteiligten Akteuren eine strategische Kultur zu etablieren, innerhalb derer sich Möglichkeiten zur Problemlösung außerhalb des situativen Kontextes von Politik ergeben. Eine Strategie bestehe dabei aus einer Problemdefinition, einem Lösungsansatz und einem Plan zur Implementation. Erfolgsbedingungen seien ein Problembewusstsein bei den beteiligten Akteuren und deren Fähigkeit zum strategischen Denken sowie die Verfügung über Macht und einen institutionellen Unterbau. Letzterer müsse für eine mediale und intellektuelle Aufmerksamkeit sorgen, denn die strategische Lösung eines gemeinsamen Problems hänge vor allem von der geteilten Perzeption der Problemmerkmale ab. Mit der Donaustrategie könne die Europäische Union genau das leisten: Sie liefere einen strategischen Entwurf für die Donauregion und ihre Akteure. Weidenfeld betrachtete die Initiative als Chance sowohl für den Donauraum als auch für die Europäische Union, denn sie könne aus den beteiligten Akteuren eine Strategiegemeinschaft formen, sei also
Die Donauraumstrategie - ein Schwerpunkt der ungarischen Ratspräsidentschaft Dr. Etelka BARSI-PATAKY, Botschafterin, Regierungsbeauftragte für die Donauraumstrategie, Budapest

Die weiteren Prioritäten der ungarischen Präsidentschaft und ihr Zusammenhang mit der Donaustrategie

S.E. Dr. József CZUKOR, Botschafter der Republik Ungarn, Berlin

Die Einbeziehung der Zivilgesellschaft in die Donaustrategie - Bestandsaufnahme und Herausforderung

Prof. Strahil KARAPCHANSKI, Abteilung Europäische Studien, Universität Ruse

Der ökologische Zustand der Donau Philip WELLER, Exekutivsekretär der Internationalen Kommission zum Schutz der Donau, Wien

Die zwischenstaatlichen, regionalen und ethnischen Spannungen im Donauraum Dr. Dušan RELJIĆ, Stiftung Wissenschaft und Politik, Berlin

Die Donau als Verkehrsader, als Wasserreservoir und als Erholungsraum

Dipl.-Ing. Hans-Peter HASENBICHLER, Geschäftsführer Via Donau, Wien

Bisherige Formen der Donaukooperation Erfolge und Defizite

Prof. Dr. Ellen BOS, Leiterin, Donau-Institut der Andrássy-Universität Budapest

Kritische Analyse der Donaustrategie Florian H. SETZEN, M.A./M.E.S., Direktor, Europa Zentrum Baden-Württemberg, Stuttgart

Die wirtschaftliche Dimension der Donaustrategie - die Rolle des privaten Sektors S.E. Dr. Lazăr COMĂNESCU, Botschafter von Rumänien, Berlin

Zusammenarbeit und Entwicklung: Bulgariens Donauperspektive

Dr. Meglena PLUGTCHIEVA, Mitglied des Bulgarischen Nationalparlaments; stellv. Ministerpräsidentin a.D.; Ruse/Sofia 
identitätsstiftend und entspreche mithin dem Gedanken der europäischen Integration.

Im folgenden Beitrag sprach Carsten Schymik über die ersten Ergebnisse der „Strategie der Europäischen Union für den Ostseeraum" (Ostseestrategie) und eröffnete den Teilnehmern und Teilnehmerinnen somit eine komparative Perspektive auf das Thema der Konferenz. Er ging auf die Entstehung, die Ziele und die Struktur der Ostseestrategie ein, verdeutlichte die Parallelen zur Donaustrategie und zeigte Unterschiede zu dieser auf. Voraussetzung für die Zustimmung aller Mitgliedstaaten zu den makroregionalen Strategien seien die drei ,Neins': Keine neuen Gesetze, keine neuen Haushaltslinien und keine neuen Institutionen für das neu zu schaffende Instrument. Laut Schymik lassen sich nach einem Jahr trotzdem Erfolge des neuen Governance-Ansatzes erkennen: So hätten sich makroregionale Netzwerke gebildet und der Diskurs der Akteure sei von einer makroregionalen Orientierung geprägt. Die Ostseestrategie habe daher einen Impetus für die regionale Zusammenarbeit bedeutet. Auf der Soll-Seite stünden die geringe Partizipation zivilgesellschaftlicher und privatwirtschaftlicher Akteure sowie die schleppende Einbeziehung von Drittstaaten wie Russland. Mangels messbarer Zielvorgaben sei zudem die Ziel-Mittel-Relation unklar, was auch dazu führe, dass der mögliche Erfolg der Ostseestrategie öffentlich kaum nachvollziehbar gemacht werden könne. Der Referent ging in seinem Vortrag zudem auf das Konzept der Makroregion ein. Die Etablierung eines solchen Raumes sei eine Möglichkeit zur Verbindung der Peripherie mit dem Zentrum. Schymik nannte weitere mögliche Makroregionen innerhalb Europas: Alpen, Atlantischer Bogen, Adria. Die Möglichkeit einer derartigen „Makroregionalisierung“ der Europäischen Union wurde im Anschluss an den Vortrag von den Teilnehmern aufgegriffen und kontrovers diskutiert.

Im dritten Vortrag stellte Michael Cramer das Projekt „Iron Curtain Trail“ vor. Dieser soll
Das Donaukompetenzzentrum - innovative Zusammenarbeit im Donautourismus

Daniela SCHILY, Projektleiterin, Deutsche Gesellschaft für Internationale Zusammenarbeit, Belgrad

Nebojša MATIJAŠEVIĆ, Projektmanager, Deutsche Gesellschaft für Internationale Zusammenarbeit, Belgrad

Die Donau: Verbindungslinie zwischen der Östlichen Partnerschaft und der EU? Die Sichtweisen Chisinaus und Kiews

Dr. Heike DÖRRENBÄCHER, Leiterin des Kiewer Büros der Friedrich-Naumann-Stiftung für die Freiheit, Kiew

als Strecke 13 in das „EuroVelo“ System, ein europaweites Netzwerk von Radwegen, integriert werden und mit seiner Route den Verlauf des Eisernen Vorhangs nachvollziehen. Cramer präsentierte Impressionen aus Natur, Geschichte und Kultur, die der „Iron Curtain Trail" nach seiner Verwirklichung vorzuweisen hätte. Die über 7.000 Kilometer lange Strecke würde im südlichen Teil ein Stück entlang der Donau verlaufen. Die Umsetzung des Projekts soll durch die überregionale $\mathrm{Zu}-$ sammenarbeit von Nichtregierungsorganisationen und lokalen Verwaltungen realisiert werden. Der „Iron Curtain Trail“" wäre somit ein Beispiel für identitätsstiftende Projekte im Rahmen übergeordneter Strategien.

Die Donaustrategie ist ein Schwerpunkt der ungarischen EU-Ratspräsidentschaft. Etelka Barsi-Pataky stellte in ihrem Vortrag die Ziele und Visionen Ungarns vor, fasste den aktuellen Stand zusammen und beschrieb die kommenden Schritte. Die Referentin nannte eine Reihe von Vorhaben: Zentral seien Infrastrukturprojekte, vor allem Straßen- und Energienetzwerke, sowie die Schaffung eines makroregionalen Forschungsrahmens. Handlungsleitend für die Umsetzung von Projekten sei die Bewahrung des Gleichgewichts zwischen der Etablierung eines fairen Wettbewerbs auf der einen Seite und dem Ausbau der sozialen Sicherungsmechanismen auf der anderen Seite. Barsi-Pataky hob die zentral- 
europäische Dimension des Jahres 2011 hervor, die sich durch die aufeinander folgenden EU-Ratspräsidentschaften von Ungarn und Polen ergäbe. „Mitteleuropäer zu sein, ist eine Stimmung", so die Referentin. Mit der Donaustrategie ergäbe sich die Chance, aus der geistigen und kulturellen Identität eine wirtschaftliche und politische Zusammenarbeit werden zu lassen. Ein starkes Mitteleuropa könne dann zu einem starken Europa beitragen.

Im ersten Vortrag des zweiten Tages der Konferenz sprach József Czukor über die Prioritäten der ungarischen Ratspräsidentschaft im Allgemeinen und über den Zusammenhang mit der EU-Donaustrategie im Speziellen. Er betonte, dass sich die Strategie für den Donauraum in den Kontext der übergeordneten Ziele der Ratspräsidentschaft einbette. Besonders gelte dies für die erste Säule der ungarischen Prioritäten „Wachstum, Arbeitsplätze und europäische Sozialpolitik" sowie für die vierte Säule „Verantwortungsvolle Erweiterung und globale Verantwortung der EU“. Die ungarische Regierung betrachte sich in ihrer Amtsausübung als ,honest broker und werde die Arbeit an Europas Hausaufgaben, der Schaffung von Finanzstabilität und wirtschaftspolitischer Koordination, weiter vorantreiben.

Strahil Karapchanski referierte zur Einbeziehung der Zivilgesellschaft in die Donaustrategie. Ein prägnantes Merkmal der Makroregion Donau sei die Heterogenität ihrer Gesellschaften und die dadurch unterschiedliche Ausprägung der Zivilgesellschaften. Das Konzept Makroregion impliziere den Einbezug zivilgesellschaftlicher Akteure in den Politikgestaltungsprozess. Wie in den offiziellen Dokumenten und von politischen Mandatsträgern betont, müsse eine Strategie auf geteilten Positionen und Werten aller Betroffenen beruhen, um erfolgreich zu sein. Nur durch Umsetzung dieses Partizipationsanspruchs könne die Zivilgesellschaft ihre Funktionen im poli- tischen Prozess erfüllen: Schaffung von Legitimation, Bereitstellung von Expertise, Kontrolle zur Sicherstellung von Effektivität und Transparenz. Karapchanski nannte mehrere Beispiele für die Umsetzung des Bottom-UpAnsatzes der Donaustrategie: die im ersten Halbjahr 2010 erfolgten Stakeholder-Konferenzen, die von der Kommission über ihre Internetplattform durchgeführte öffentliche Konsultation sowie das von der Stiftung ,Foster Europe“ organisierte „Danube Basin NGO Network Project“. Ein Kritikpunkt sei der für einige Akteure eingeschränkte Zugang zu den Konsultationsprozessen aufgrund mangelnder Vielsprachigkeit und hoher Kosten für die Anreise. Auch infolge dieser Gegebenheiten seien die Foren durch stärker professionalisierte Organisationen dominiert. Im Fazit betonte Karapchanski, dass erst durch den Einbezug der Zivilgesellschaft die Donaustrategie zu einem Instrument der Europäisierung werde.

Der ökologische Zustand der Donau war Thema des folgenden Beitrags von Philip Weller. Der Referent veranschaulichte die Bedeutung der Donau als ,vielfältigen Lebensraum vom Schwarzwald bis zum Schwarzen Meer" und beschrieb die historische Umweltbelastung im Donauraum durch Abholzung, Staudämme und Pestizideinleitung. Seit der Unterzeichnung des „Übereinkommens über die Zusammenarbeit zum Schutz und zur verträglichen Nutzung der Donau (Donauschutzübereinkommen)“4 im Jahr 1994 habe sich viel getan. Es gebe Initiativen zur Beschränkung der Gefahrenstoff- und Pestizidbelastung und zur Beseitigung von Hindernissen für die Wanderung von Fischen. Weller wies darauf hin, dass Umweltmanagement nur unter Berücksichtigung des gesamten Einzugsgebiets, also auch der Regionen an den Nebenflüssen der Donau, erfolgreich sein könne. Ein transnationales Monitoring Netzwerk für die Wasserqualität, ein Unfallmanagementund ein Vorwarnsystem für den Hochwasserschutz seien bereits vorhanden und müssten 
nicht neu geschaffen werden. Den Mehrwert der Donaustrategie erkannte Weller in der Integration der Bereiche Umweltschutz, Wirtschaft und Verkehr und in der Generierung von politischer und finanzieller Unterstützung für die Projekte der Internationalen Kommission zum Schutz der Donau.

Die zwischenstaatlichen, regionalen und ethnischen Spannungen im Donauraum bildeten den Gegenstand des Beitrags von Dušan Reljić. Ausgangspunkt des Referates war die Feststellung, dass die Formulierungen in den offiziellen Dokumenten der Donaustrategie an der Zweiteilung in ein ,zivilisiertes“ Westeuropa und ein ,unterentwickeltes“ Südosteuropa und an der Mystifizierung der Region festhalten würden. Die Europäische Union perzipiere Südosteuropa noch immer unter den Aspekten Sicherheit, Abwehr von Bedrohungen und Import von Instabilität. Darauf aufbauend formulierte Reljić die Leitfrage seines Vortrags: Kann die Europäische Union zur Lösung der in der Region vorhanden Konflikte beitragen? Im Folgenden skizzierte er das komplizierte Geflecht der Konfliktlinien in der Region mit Bezug auf deren ethnische, nationale und historische Dimensionen. Das in einigen Bereichen vorherrschende Prinzip des Nationalismus und die damit verbundene politische Kultur benannte Reljić als einen der großen Stolpersteine für Lösungsprozesse. Die schwelenden Konflikte zwischen den Kandidatenstaaten müssten zwingend noch vor deren EU-Beitritt gelöst werden. Die Europäische Union könne dann eine Antwort auf die in der Region vorhandenen Spannungen sein, wenn die Idee der kulturellen Heterogenität Teil des europäischen Integrationsprozesses bleibe und die Europäische Union nicht auf die Bereiche Handel und Sicherheit beschränkt werde.

„Via Donau“ nimmt im Auftrag der Österreichischen Republik öffentliche Aufgaben in den Bereichen Hochwassermanagement, Logistik und Infrastruktur entlang der Donau wahr. Hans-Peter Hasenbichler präsentierte die Arbeit der Wasserstraßengesellschaft und stellte die Donau als Querschnittsmaterie und in ihrer Nutzungs- und Funktionsvielfalt dar. Der Referent beschrieb ökonomische und ökologische Vorteile der Binnenschifffahrt gegenüber anderen Transportwegen und illustrierte den Stand der Entwicklung hin zur Donau als einem leistungsfähigen Verkehrsweg. Der Ausbau der Häfen zu multimodalen Logistik-Knotenpunkten spiele dabei eine zentrale Rolle. Straße, Schiene und Wasserweg stünden wegen gegenseitiger Abhängigkeit nicht in Konkurrenz zueinander. Eine Verbesserung der Umweltperformance könne durch eine Modernisierung der Donau-Flotte weiter vorangetrieben werden. Projekte, die Ökologie und Ökonomie integrieren, stellten bereits jetzt die Mehrzahl der von „Via Donau“ koordinierten Initiativen dar. Den Mehrwert der Donaustrategie sieht Hasenbichler im stärkeren Engagement der Anrainer und in der durch die Strategie erfolgten Prioritätenreihung.

Ellen Bos referierte zu den Erfolgen und Defiziten bisheriger Formen der Donaukooperation. Ihr historischer Rückblick reichte von den Zeiten des Römischen Imperiums über die des Habsburger Reiches bis zu den heutigen Ereignissen. In der Geschichte habe die Funktion der Donau immer wieder zwischen Grenze und Verbindung gewechselt. Eine regionsübergreifende Identität hätte es bei den bisherigen Kooperationsformen nicht gegeben, die Idee eines Donauraumes existiere jedoch schon länger. In den letzten Jahren hätten die neuen Prioritäten, Umweltschutz und Wassermanagement, in den nationalen Politiken zu einer Vertiefung der regionalen Kooperation geführt. Die Donaustrategie sei im Kontext dieser neuen Entwicklungen zu betrachten und könne zur Bildung einer regionalen Identität beitragen.

In einer kritischen Analyse setzte sich Florian Setzen mit dem Kommissionsentwurf zur Donaustrategie auseinander. Der Referent vollzog den Entstehungsprozess des Dokumentes nach und analysierte die Inhalte des Strategieentwurfs. Bezüglich der sachpolitischen In- 
halte fiele auf, dass kritische Themen wie Korruption und der Umgang mit Minderheiten ausgeklammert werden. Darüber hinaus würden Ziele, die den vier allgemeinen Strategieprioritäten zugeordnet sind, nicht definitiv festgelegt, sondern mit dem Attribut „möglich" versehen. Die Verbindlichkeit des Dokuments würde dadurch verringert, die Effektivitätsmessung erschwert. Bezüglich der Inhalte zur politischen Steuerung und Umsetzung konstatierte Setzen, dass die Governance-Strukturen vieles im Unklaren ließen und $\mathrm{zu}$ Kompetenzüberschneidungen führen würden. So sei nicht geklärt, ob die Programmkoordinatoren eine Weisungsbefugnis hätten und wie das Verhältnis der nationalen Kontaktstellen zu den Programmkoordinatoren sei. Der Referent bezweifelte, dass es einen großen Andrang zwischenstaatlicher Organisationen geben werde, die sich um die Funktion der Projektkoordination bewerben, da diese Rolle zwar zu mehr Verantwortung und Arbeit nicht aber zu größerer Entscheidungskompetenz und zusätzlichen Fördermitteln führen würde. Schlussendlich würden sich die Aufgaben der Kommission - Vermittlung, Unterstützung, Evaluation - mit denen der Projektkoordination überschneiden. Trotz der genannten Kritikpunkte kam Setzen zu der Einschätzung, dass die Strategie als Referenzpapier und als Grundlage zur Selbstbeurteilung der Fortschritte der beteiligten Staaten Verwendung finden könne. Steuerung könne vor allem durch die Prioritätenordnung und den neuen Länderzuschnitt der Programme erreicht werden.

Lazăr Comănescu bezeichnete die Donaustrategie als große Chance für Rumänien. In seinem Vortrag ging es um die wirtschaftliche Dimension der Strategie und um die Rolle des privaten Sektors. Der Referent sprach vom Leben von und mit der Donau in Rumänien. Zum einen fließe diese über 1.000 Kilometer durch das Land, zum anderen mündeten fast alle Flüsse des Landes in diesen Strom. Das Einzugsgebiet der Donau spiele bei Energiegewinnung, Landwirtschaft und Verkehr eine zentrale Rolle - Initiativen zur Einbindung privatwirtschaftlicher Akteure könnten in diesen Bereichen ansetzen. Als vielversprechenden Ansatz sah Comănescu das „Bucharest Business Forum“ vom Oktober 2010. Wichtig sei die Modernisierung der 30 Binnenhäfen entlang der Donau sowie der Ausbau des Hafens in Konstanza, der zu den zehn größten Hochseehäfen Europas gehöre. Der Referent beendete seinen Beitrag mit einem Aufruf an deutsche Unternehmen, sich an den geplanten Projekten zu beteiligen.

Meglena Plugtchieva teilte den Optimismus ihres Vorredners. In ihrem Vortrag zu Bulgariens Donauperspektive illustrierte sie die Bedeutung der Strategie für den Ausbau der paneuropäischen Verkehrskorridore. Auch der Ausbau der Energieversorgungsnetze, Umweltschutz, Überschwemmungsprävention und Tourismusmanagement ständen auf der Prioritätenliste der bulgarischen Regierung. Auf der 470 Kilometer langen Flussgrenze zwischen Bulgarien und Rumänien gäbe es bisher nur eine Brücke, eine zweite sei im Entstehen. Brückenbau, auch im übertragenen Sinne, werde mithilfe der Europäischen Union in den nächsten Jahren vorangetrieben.

In dem Beitrag von Daniela Schily und $\mathrm{Ne}$ bojša Matijašević ging es um die Arbeit des „Donaukompetenzzentrums“ (DCC) und um innovative Zusammenarbeit im Donautourismus. Das volle Potenzial des Donautourismus könne sich bisher nicht entfalten, da der obere Flussabschnitt als „Tourismusdestination mit 2000 km Wurmfortsatz" betrachtet werde, die untere Donau für den Tourismus also keine Rolle spiele. In dem Projekt „Danube Goes Beyond" versuche das von der Deutschen Gesellschaft für Internationale Zusammenarbeit (GIZ) unterstützte DCC deshalb, die untere Donau als Marke einzuführen und für Touristen interessant zu machen. Zur Umsetzung müssten die nationalen Politiken zu einer internationalen Vermarktungsstrategie ausgebaut, die touristische Infrastruktur verbessert und das Stigma der politischen Instabilität überwunden werden. Diese Ziele fänden direkt oder indirekt auch in der Donaustrategie 
Erwähnung. Der Begriff Donau müsse Assoziationen wie Naturschönheit, Gastfreundlichkeit und kultureller Reichtum wecken. Das Vorhandensein dieser Merkmale in der Region zeigte die Präsentation eindringlich.

Im letzten Vortrag der Veranstaltung stellte Heike Dörrenbächer die Sichtweisen zweier Länder vor, die zwar Partner in der Donauraumstrategie, nicht aber Mitglieder der Europäischen Union sind: die der Ukraine und der Republik Moldau. Der Machtwechsel in ersterer habe zu einer Rückkehr zur Multivektorenpolitik geführt, einer unstetigen Außenpolitik deren Orientierung zwischen Europa und Russland wechselt. In der Ukraine gebe es eine autoritäre Entwicklung und eine Abkehr von den Werten und Prinzipien der Europäischen Union. Der Schwerpunkt der Donaustrategie auf projektbezogener und damit pragmatischer Zusammenarbeit sei insofern förderlich, als dass diese gut zur ukrainischen Politik der selektiven Modernisierung passe. Ins Auge fielen hier vor allem Infrastrukturvorhaben wie Tiefwasserkanäle und Autobahnabschnitte. Da es keine neuen Gelder gäbe, sei der Anreiz zur Zusammenarbeit für die ukrainische Regierung aber gering. Ganz anders sei die Situation in der Republik Moldau, in der die (Parteien-)Allianz für die Europäische Integration die EU-Mitgliedschaft als Ziel formuliert habe. Dieser gehe es nicht nur um Energiesicherheit und die Anbindung an europäische Transportnetze, sondern auch um die Anpassung an europäische Werte und Standards. Die Donaustrategie könne somit zu einem „Entwicklungsprogramm“ für das gesamte Land werden. In beiden Staaten werde die Strategie nur zum Erfolg führen, wenn sie seitens einflussreicher Akteure in der Europäischen Union starke politische Unterstützung erhalte.

\section{Ein grundlegender Beitrag über ein Randgebiet der europäischen Einigung}

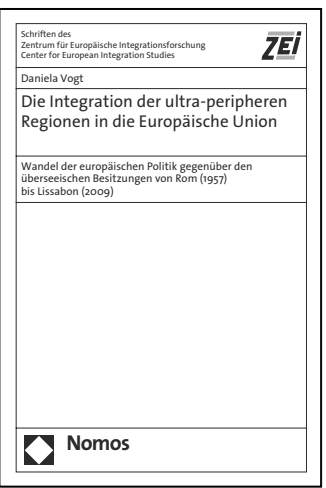

\section{Die Integration der ultra-peripheren Regionen in die Europäische Union} Wandel der europäischen Politik gegenüber den überseeischen Besitzungen von Rom (1957) bis Lissabon (2009)

Von Dr. Daniela Vogt, M.A.

2011, 235 S., geb., 49, $-€$

ISBN 978-3-8329-6580-8

(Schriften des Zentrum für Europäische

Integrationsforschung (ZEI), Bd. 75)

nomos-shop.de/13586

Bitte bestellen Sie im Buchhandel oder versandkostenfrei unter $\downarrow$ www.nomos-shop.de

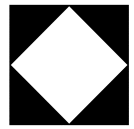

Nomos 